\title{
Characteristics of strong ferromagnetic Josephson junctions with epitaxial barriers
}

\author{
C. Bell* \\ Materials Science Department, IRC in Superconductivity and IRC in Nanotechnology, University of Cambridge, Cambridge, UK \\ R. Loloee \\ Department of Physics and Astronomy, Center for Sensor Materials and Center for Fundamental Material Research, \\ Michigan State University, East Lansing, Michigan 48824, USA \\ G. Burnell, M. G. Blamire \\ Materials Science Department, IRC in Superconductivity and IRC in Nanotechnology, University of Cambridge, Cambridge UK
}

(Dated: November 5, 2018)

\begin{abstract}
We present the measurement of superconductor / ferromagnetic Josephson junctions, based on an epitaxial $\mathrm{Nb}$ bottom electrode and epitaxial $\mathrm{Fe}_{20} \mathrm{Ni}_{80}$ barrier. Uniform junctions have been fabricated with a barrier thicknesses in the range $2-12 \mathrm{~nm}$. The maximum critical current density $\sim 2.4 \pm 0.2 \times 10^{9} \mathrm{Am}^{-2}$ was found for a devices with a $3 \mathrm{~nm}$ thick barrier at $4.2 \mathrm{~K}$, corresponding to an average characteristic voltage $I_{C} R_{N} \sim 16 \mu \mathrm{V}$. The $I_{C} R_{N}$ showed a non-monotonic behavior with $\mathrm{Fe}_{20} \mathrm{Ni}_{80}$ thickness. The variation of the resistance of a unit area $A R_{N}$, of the junctions with barrier thickness gave a $\mathrm{Nb} / \mathrm{Py}$ specific interface resistance of $6.0 \pm 0.5 \mathrm{f} \Omega \mathrm{m}^{2}$ and $\mathrm{Fe}_{20} \mathrm{Ni}_{80}$ resistivity of $174 \pm 50 \mathrm{n} \Omega \mathrm{m}$, consistent with other studies in polycrystalline samples.

PACS numbers: 74.45.+c, 72.25.Mk, 75.70.Cn

Keywords: Josephson junction, ferromagnet, epitaxial
\end{abstract}

\section{INTRODUCTION}

The early research into the proximity effect between superconductors $(\mathrm{S})$ and ferromagnets $(\mathrm{F})$ concentrated on measurements of the critical temperature $T_{C}$ and critical field of $\mathrm{S} / \mathrm{F}$ heterostructures. A motivating factor for this research was the realization of the $\pi$ state, in which the groundstate phase difference between S layers was changes from 0 to $\pi$, due to the oscillation of the superconducting order parameter induced in the $\mathrm{F}$ layer $\stackrel{1}{*}$ The transition should manifest itself as a nonmonotonic change in the properties of the multilayers, as a function of $\mathrm{F}$ layer thickness $d_{F}$. Many epitaxial and polycrystalline systems involving different materials were investigated, using various growth techniques. Although oscillatory $T_{C}\left(d_{F}\right)$ were observed in several experiments (see reference ${ }^{2}$ for a review), these studies were complicated by interface effects and 'dead' magnetic layers at the $\mathrm{S} / \mathrm{F}$ interface which made the interpretation of the oscillatory $T_{C}$ more difficult $\underline{\underline{3}}$

It was not until the measurement of current perpendicular to plane (CPP) Josephson junctions with ferromagnetic barriers $(\mathrm{S} / \mathrm{F} / \mathrm{S})$ that the $\pi$-shift could be conclusively demonstrated. Such $\pi$-junctions have been characterised as a function of temperature and $d_{F}, \frac{4.5}{4}$ using alloys whose composition could be tuned to achieve an appropriately low Curie temperature $\left(T_{M}\right)$, such that the $0-\pi$ crossover could be observed in a easily realizable window of experimental phase space. These $\pi$-junctions have since been incorporated into various loop geometries to further demonstrate the $\pi$ shift 6.7

$\mathrm{S} / \mathrm{F} / \mathrm{S}$ junctions have also been fabricated with the relatively high $T_{M}$ ferromagnets $\mathrm{Ni}_{2}, \frac{8}{2} \mathrm{Co}, \frac{9}{2}$ and composite $\mathrm{Co} / \mathrm{Cu} / \mathrm{Fe}_{20} \mathrm{Ni}_{80}$ structures ${ }^{10}$ In these cases the junc- tions are much more sensitive to the barrier properties and $d_{F}$, and hence the $0-\pi$ crossover has not been demonstrated in junctions with strong ferromagnetic barriers.

In all of the above CPP junctions, the $\mathrm{S}$ and $\mathrm{F}$ layers have been polycrystalline and in the dirty limit. The realization of epitaxial junctions in the clean limit may remove some of the difficulties of measuring low $T_{M}$ alloy systems, (which are sensitive to stoichiometry and harder to characterize magnetically), as well as the sensitivity to $d_{F}$ of the high $T_{M}$ barriers. Clean limit junctions are also expected to show a novel non-sinusoidal currentphase relationship ${ }^{11}$, in contrast to recent measurements of $\mathrm{S} / \mathrm{F} / \mathrm{S}$ junctions with $\mathrm{Cu}_{x} \mathrm{Ni}_{1-x}$ alloy barriers ${ }^{12}$

$\mathrm{S} / \mathrm{F} / \mathrm{S} \pi$-junctions have been proposed as potential logic elements in quantum computing circuits 13,14 ; however the present critical current - resistance product $\left(I_{C} R_{N}\right)$ values are relatively small at present. The clean limit may again provide a route to achieving much higher critical current densities $J_{C}$, and hence $I_{C} R_{N}$. The combination of all of these factors motivates the investigation of $\mathrm{S} / \mathrm{F} / \mathrm{S}$ Josephson junctions based on epitaxial ferromagnetic barriers. The epitaxial $\mathrm{Fe}_{20} \mathrm{Ni}_{80}(\mathrm{Py})$ system is also of interest in spintronic applications, such as the fabrication of ballistic spin valves and spin torque devices, and also provides an interesting comparison to the previous studies of polycrystalline $\mathrm{Nb} /$ normal metal $(\mathrm{N})$ and $\mathrm{F} / \mathrm{N}$ interfaces (for example ${ }^{15,16,17}$ ). 


\section{FILM GROWTH AND CHARACTERIZATION}

Our $(1 \overline{1} 0) \mathrm{Nb} /(111) \mathrm{Fe}_{20} \mathrm{Ni}_{80}$ (Py) films are grown by sputtering on $(11 \overline{2} 0) \quad \mathrm{Al}_{2} \mathrm{O}_{3}$ as described in detail elsewhere 18 To improve the epitaxy and reduce the strain in the films, the actual device structure is $\mathrm{Nb} / \mathrm{Cu} / \mathrm{Py} / \mathrm{Cu} / \mathrm{Nb}$ with the thicknesses of the two $\mathrm{Cu}$ layers $\sim 5 \mathrm{~nm}$, (with (111) orientation). The $\mathrm{Cu}$ layers are expected to be strongly proximitized by the $\mathrm{Nb}$ electrodes and should not significantly reduce the $J_{C}$ of the devices. The Py thickness $d_{\mathrm{Py}}$, was in the range $2-12$ $\mathrm{nm}$. The samples with $d_{\mathrm{Py}}=2,4$ and $6 \mathrm{~nm}$ were initially grown with a bottom $\mathrm{Nb}$ layer of thickness 200 $\mathrm{nm}$, and a top electrode of $20 \mathrm{~nm}$ of $\mathrm{Nb}$ followed by a $5 \mathrm{~nm} \mathrm{Au}$ capping layer. This $\mathrm{Au}$ was removed ex-situ by $\mathrm{Ar}$ ion milling, and the top $\mathrm{Nb}$ electrode deposited by further d.c. sputtering. All of the remaining samples were grown in a second deposition, with the top and bottom $\mathrm{Nb}$ thicknesses of $\sim 250 \mathrm{~nm}$, deposited in-situ. To achieve epitaxy, the Py barrier was grown at $423 \mathrm{~K}$, and the $\mathrm{Nb}$ at $1023 \mathrm{~K}$. It was not possible therefore to grow the top Nb electrode epitaxially at the reduced temperatures required for the $\mathrm{Cu}$ and Py layers. Fig. 四shows the

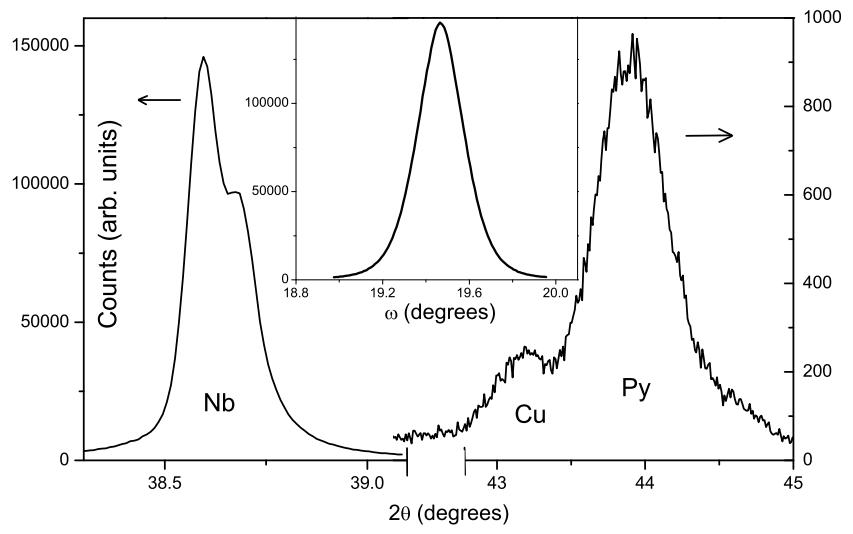

FIG. 1: X-ray diffraction scan of $(1 \overline{1} 0) \mathrm{Nb},(111) \mathrm{Cu}$ and (111) Py peaks at positions $2 \theta=38.6,43.2$ and $43.9^{\circ}$ respectively. The splitting of the $\mathrm{Nb}$ peak is caused by the presence of the two $\mathrm{CuK}_{\alpha}$ radiation lines. Inset: An $\omega$ scan of the primary $\mathrm{Nb}$ peak.

x-ray diffraction peaks of the $\mathrm{Nb}, \mathrm{Cu}$ and Py layers in the $d_{\mathrm{Py}}=6 \mathrm{~nm}$ sample, (taken with $\mathrm{CuK}_{\alpha}$ radiation using a Philips X'Pert powder diffractometer). The full width half maximum values obtained from $\omega$ scans were $\sim 0.25^{\circ}$ for the $\mathrm{Nb}$ layer (inset of Fig. (1), and $\sim 0.78^{\circ}$ for the $\mathrm{Py}$ layer (not shown). This confirms the epitaxial nature of the bottom electrode and barrier. A resistance vs temperature, $R(T)$, measurement of the unpatterned $d_{\mathrm{Py}}=2$ $\mathrm{nm}$ film (before further $\mathrm{Nb}$ was deposited, such that the relatively thick epitaxial $\mathrm{Nb}$ layer dominates the conductivity) was also made. The film showed a residual resistance ratio $R(T=10 \mathrm{~K}) / R(T=300 \mathrm{~K})=14.2$. This is of similar order to other epitaxial Nb films, in which the su- perconducting coherence length $\xi_{S}=18 \mathrm{~nm},{ }^{19}$ compared to a typical dirty limit value of $\sim 6 \mathrm{~nm}$ in polycrystalline sputtered films ${ }^{20.21}$

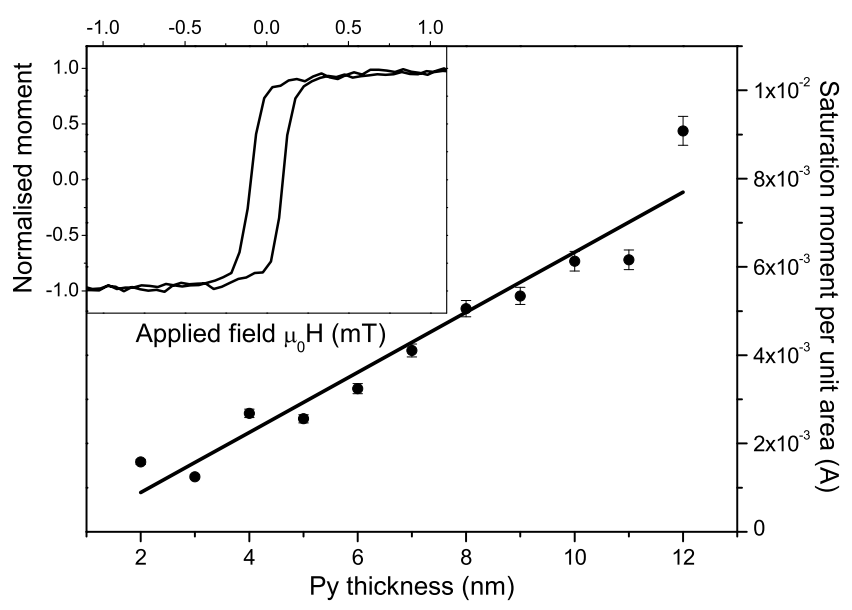

FIG. 2: Scaling of the magnetic moment per unit area vs Py thickness. The line is a least squares fit to the data. Inset: Hysteresis loop of the $2 \mathrm{~nm}$ Py film at $295 \mathrm{~K}$.

The magnetic properties were characterized with a vibrating sample magnetometer at room temperature. Fig. 2 shows the saturation moment per unit area of the films in this study, (along with a typical hysteresis loop shown in the inset). Extrapolating the least square fit gives a nominal magnetically 'dead' layer of thickness $\sim 7 \AA$.

The films were patterned using optical lithography, followed by broad beam Ar ion milling $\left(1 \mathrm{mAcm}^{-2}, 500 \mathrm{~V}\right)$ to micron scale wires and associated tracks and contact pads, to allow four point measurements to be performed on the devices. These tracks were then processed in a $\mathrm{Ga}^{+}$focused ion beam to achieve vertical transport with a device area in the range $0.05-1.1 \mu \mathrm{m}^{2}$. This fabrication process is described in detail elsewhere ${ }^{22}$ and has been used previously to fabricate Josephson junctions with strong ferromagnetic barriers $\frac{10}{10}$ Transport measurements were made in a liquid He dip probe. The differential resistance as a function of bias current of the junction was made with a lock-in amplifier, and the $I_{C}$ found using a resistive criterion. The $R_{N}$ was measured using a quasi-d.c. bias current of $3-5 \mathrm{~mA}$. This enabled the non-linear part of the $I-V$ near to $I_{C}$ to be neglected, but was not too large to drive the $\mathrm{Nb}$ electrodes normal.

\section{RESULTS AND DISCUSSION}

An $I_{C}(H)$ modulation obtained in a junction with lateral area $\sim 1060 \mathrm{~nm} \times 250 \mathrm{~nm}$ is shown in Fig. 3 In this case the field is applied in the direction perpendicular to the larger dimension of the device. It is expected that the coercive field of the Py should increase relative to that taken from the room temperature hysteresis loop shown in Fig. 2] due to the reduced temperature and the 
aspect ratio of the sub-micron patterned device. ${ }^{23}$ In this case the applied field required to modulate the $I_{C}$ does not significantly affect the magnetization of the $\mathrm{Py}$, and the $I_{C}(H)$ is symmetric about zero field. The good fit to the ideal 'Fraunhofer' pattern indicates a uniform current flow in the junction. Relatively smaller junctions, which require larger fields to modulate the $I_{C}$, were found to show hysteresis which we associate with changes in the Py domain structure and magnetization. Net magnetic induction present in the barrier is known to shift the $I_{C}(H)$ pattern away from $H=0$ and reduce the $I_{C}$ at zero field from its true maximum value ${ }^{24}$ To avoid falsely

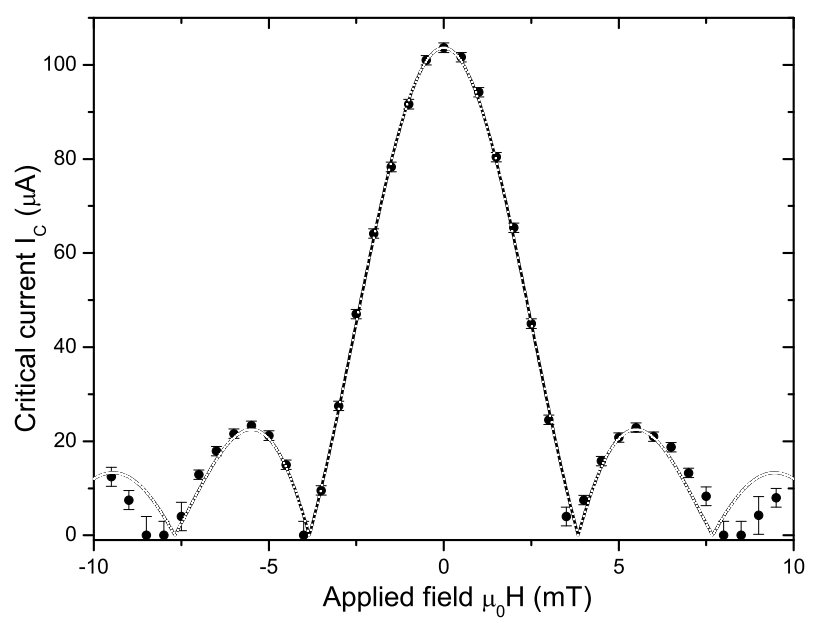

FIG. 3: $I_{C}(H)$ modulation for a device demagnetized at $4.2 \mathrm{~K}$ for a $2 \mathrm{~nm}$ thick Py barrier. Device dimension in the direction perpendicular to the applied field $\sim 1.06 \mu \mathrm{m}$. Line is a best fit to a 'Fraunhofer' function.

small values of $I_{C}$, the films were demagnetized at 4.2 $\mathrm{K}$, as well as the $I_{C}(H)$ pattern being directly measured where possible, (for devices with $I_{C} R_{N}>2 \mu \mathrm{V}$ ). In some cases however, the offset of the maximum $I_{C}$ from $H=0$ could not be removed. The cause of this is ascribed to shape anisotropy in the sub-micron devices which provides an additional demagnetizing field which may prevent the formation of a flux-closed domain structure in the barrier.

The variation of $I_{C} R_{N}\left(d_{\mathrm{Py}}\right)$ is shown in Fig. 4 Other devices showed much larger $J_{C}$ values, but showed strongly distorted or no $I_{C}(H)$ modulation, implying shorting around the edges of the junctions due to redeposited material during device fabrication. For $d_{\mathrm{Py}}>8$ $\mathrm{nm}$ the devices showed some reduction of the differential resistance around zero current bias, but did not show a measurable supercurrent at $T=4.2 \mathrm{~K}$, (this was also the case in several devices with $d_{\mathrm{Py}}=7$ and $8 \mathrm{~nm}$ ). No re-entrant $I_{C} R_{N}$ was observed up to $d_{\mathrm{Py}}=12 \mathrm{~nm}$. It is clear that there is a strong suppression of $I_{C} R_{N}$ for increasing $d_{\mathrm{Py}}$, but despite some scatter in the data of Fig. 4 for each set of devices with constant $d_{\mathrm{Py}}$, the decay is clearly not purely exponential. A component of

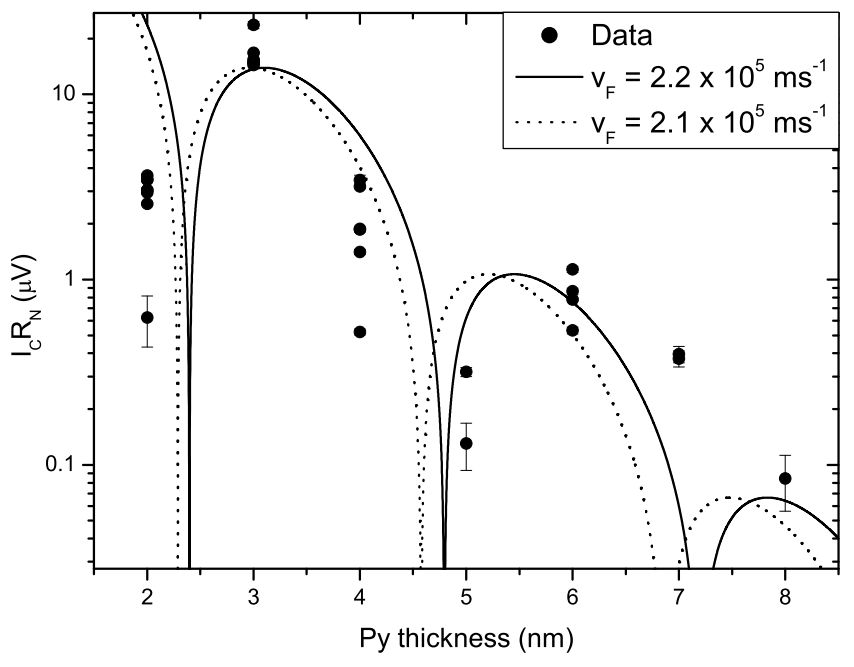

FIG. 4: Characteristic voltage $I_{C} R_{N}$, as a function of Py thickness at $T=4.2 \mathrm{~K}$. Dashed and solid lines are two fits to Eq. (1), as described in the text.

this non-monotonic change may be associated with the slightly different preparation methods of the 2,4 and 6 $\mathrm{nm}$ thick barriers, or run to run variation in the system. For example for $d_{\mathrm{Py}}=3 \mathrm{~nm}$, the fully in-situ deposition may imply a higher quality and larger $I_{C} R_{N}$, however this is inconsistent with the same comparison between the samples with $d_{\mathrm{Py}}=4,5$ and $6 \mathrm{~nm}$. Therefore the variation in $I_{C} R_{N}$ would seem to be a true effect associated with the oscillatory induced superconducting order parameter in the Py layer.

We can compare the behavior of these junctions to

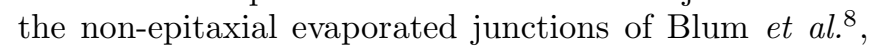
with the structure $\mathrm{Nb} / \mathrm{Cu} / \mathrm{Ni} / \mathrm{Cu} / \mathrm{Nb}$. In that case a measurable critical current at $T=4.2 \mathrm{~K}$ was observed up to a Ni thickness of $9 \mathrm{~nm}$ - similar to the present data. Due to the relatively small number of data points and the scatter, it is difficult to accurately fit this non-monotonic decay. We model the data using

$$
I_{C} R_{N} \propto\left|\sin \left(2 E_{\mathrm{ex}} d_{F} / \hbar v_{F}\right)\right| /\left(2 E_{\mathrm{ex}} d_{F} / \hbar v_{F}\right),
$$

where $v_{F}$ is the Fermi velocity of $\mathrm{Py}$, and $E_{\mathrm{ex}}$ the exchange energy. ${ }^{1,8}$ These two parameters have recently been measured 25 in $\mathrm{Py}$ as $E_{\mathrm{ex}}=135 \mathrm{meV}$ and $v_{F}=$ $2.2 \pm 0.2 \times 10^{5} \mathrm{~ms}^{-1}$ (for the majority spin). The lines in Fig. 4 correspond to $E_{\mathrm{ex}}=95 \mathrm{meV}$, with both $v_{F}=2.2$ and $2.1 \times 10^{5} \mathrm{~ms}^{-1}$, to indicate the degree of variation the error in $v_{F}$ causes. The agreement between the data and the model is not ideal, however for an order of magnitude estimate it is clear that the fit is acceptable. The period of oscillation of $I_{C} R_{N}\left(d_{F}\right)$ is given by $\pi \hbar v_{F} / E_{\mathrm{ex}}$, and can therefore be estimated to be of the order of $5 \mathrm{~nm}$, again similar in magnitude to the $5.4 \mathrm{~nm}$ value obtained by Blum et $a l^{8}$.

For the previously mentioned $\mathrm{Ni}$ junctions 8 , a maximum $I_{C} \sim 20 \mathrm{~mA}$ was observed at $T=4.2 \mathrm{~K}$ in $10 \times 10 \mu \mathrm{m}^{2}$ devices for a $1 \mathrm{~nm}$ thick Ni barrier, giv- 
ing $J_{C}=2 \times 10^{8} \mathrm{Am}^{-2}$. In our samples, one device with $d_{\mathrm{Py}}=3 \mathrm{~nm}$ had $I_{C}=1.03 \mathrm{~mA}$ with a lateral area of $0.35 \pm 0.02 \mu \mathrm{m}^{2}$, giving the highest $J_{C} \sim 2.9 \pm 0.2 \times 10^{9}$ $\mathrm{Am}^{-2}$ at the same temperature, the average for $d_{\mathrm{Py}}=3$ $\mathrm{nm}$ was $J_{C}=2.4 \pm 0.2 \times 10^{9} \mathrm{Am}^{-2}$. The larger $J_{C}$ for the epitaxial films can be attributed to the increased $\xi_{S}$ of the $\mathrm{Nb}$ bottom electrode.

Finally, we have measured the total resistance $\left(R_{N}\right)$ of a unit area $A R_{N}$, of all junctions. The variation of $A R_{N}$ for all devices vs. $d_{\text {Py }}$ over the range of $2 \mathrm{~nm}<$ $d_{\text {Py }}<12 \mathrm{~nm}$ are shown in Fig. 5] Here $A R_{N}$ is the total specific resistances; consisting of the specific resistance of the $\mathrm{S} / \mathrm{F}$ interfaces $\left(A R_{\mathrm{Nb} / \mathrm{Py}}\right)$ in the $\mathrm{S} / \mathrm{F} / \mathrm{S}$ sandwich and the ferromagnetic layer $\stackrel{15}{1}$ such that

$$
A R_{N}=2 A R_{\mathrm{Nb} / \mathrm{Py}}+\rho_{\mathrm{Py}} d_{\mathrm{Py}} .
$$

If we exclude one of the data points (with the highest $A R_{N}$ for $d_{\mathrm{Py}}=2 \mathrm{~nm}$ ), the best fit straight line to our data gives an ordinate intercept of $2 A R_{\mathrm{Nb} / \mathrm{Py}}=6.0 \pm 0.5$ $\mathrm{f} \Omega \mathrm{m}^{2}$ and the resistivity $\rho_{\mathrm{Py}}=174 \pm 50 \mathrm{n} \Omega \mathrm{m}$ (from the slope). The values fall within the range of $2 A R_{\mathrm{Nb} / \mathrm{Py}}=$ $6-7.5 \mathrm{f} \Omega \mathrm{m}^{2}$ and $\rho_{\mathrm{Py}}=110-140 \mathrm{n} \Omega \mathrm{m}$ reported for polycrystalline samples elsewhere $\underbrace{15.17}$

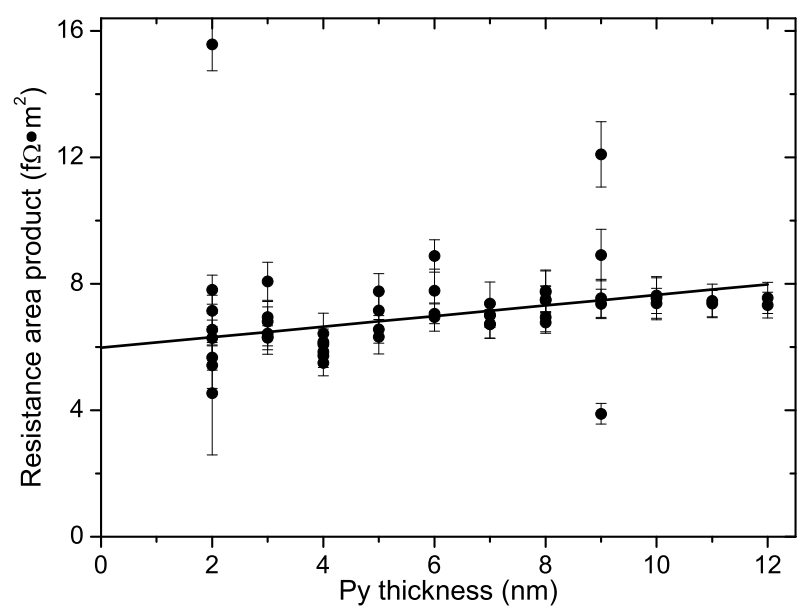

FIG. 5: Resistance area product $\left(A R_{N}\right)$ at $T=4.2 \mathrm{~K}$ as a function of Py thickness. Line is a best fit excluding the point with the largest $A R_{N}$ for $d_{\mathrm{Py}}=2 \mathrm{~nm}$.

\section{SUMMARY}

We have measured the Josephson current through a epitaxial Py barrier and observed high quality junction characteristics. The $I_{C} R_{N}\left(d_{\mathrm{Py}}\right)$ showed a non-monotonic behavior, which could be approximately modelled with a simple model. We observed no sign of re-entrant behavior above $d_{\mathrm{Py}}=8 \mathrm{~nm}$. The data extracted from the $A R_{N}\left(d_{\mathrm{Py}}\right)$ product was consistent with polycrystalline samples.

While interesting for spin-valve junctions, $\mathrm{Py}$ is not an ideal material in which to fully explore the properties of ferromagnetic Josephson junctions in the clean limit since the spin diffusion length is relatively short.16.25 A reduced spin diffusion length can have a strong influence on the possible realization of $\pi$-junctions formed by the interference of multiple Andreev reflection processes at the $\mathrm{S} / \mathrm{F}$ interfaces. The rôle of different reflection amplitudes for the minority and majority spins, and the spin polarisation have been considered elsewhere. ${ }^{26,27}$ Other effects on the Josephson current due to shape of the Fermi surface in the epitaxial barrier may also be a consideration, in an analogous fashion to the requirements in tunnel junctions 28 The use of elemental, or other epitaxial F layers - with longer spin diffusion lengths - may allow even further increases of the $J_{C}$ to be achieved in these structures, and rule out any problems associated with a loss of spin memory. With a suitable choice of material and growth technique, the fully epitaxial $\mathrm{S} / \mathrm{F} / \mathrm{S}$ would also be an important system to study.

\section{ACKNOWLEDGEMENTS}

We thank J. Aarts for pointing out the importance of the spin diffusion length in these systems, as well as E. J. Tarte, C. W. Leung, W.P. Pratt and J. Bass for useful discussions and assistance. We acknowledge the support of the Engineering and Physical Sciences Research Council UK, the U.S. NSF grant 98-09688 and the European Science Foundation $\pi$-shift network.
* Present address: Kamerlingh Onnes Laboratory, Universiteit Leiden, Leiden, The Netherlands. Email: bell@physics.leidenuniv.nl

1 A. I. Buzdin, L. N. Bulaevskiı̌, and S. V. Panyukov, Pis'ma Zh. Éksp. Teor. Fiz. 35, 147 (1982), [JETP Lett. 35178 (1982)].

2 Y. A. Izyumov, Y. N. Proshin, and M. G. Khusainov, Usp. Fiz. Nauk 172, 113 (2002), [Phys. Usp. 45109 (2002)].

3 J. Aarts, J. M. E. Geers, E. Brück, A. A. Golubov, and R. Coehoorn, Phys. Rev. B 56, 2779 (1997).
${ }^{4}$ V. V. Ryazanov, V. A. Oboznov, A. Y. Rusanov, A. V. Veretennikov, A. A. Golubov, and J. Aarts, Phys. Rev. Lett. 86, 2427 (2001).

5 T. Kontos, M. Aprili, J. Lesueur, and X. Grison, Phys. Rev. Lett. 86, 304 (2001).

${ }^{6}$ V. V. Ryazanov, V. A. Oboznov, A. V. Veretennikov, and A. Y. Rusanov, Phys. Rev. B 65, 020501(R) (2001).

7 A. Bauer, J. Bentner, M. Aprili, M. L. D. Rocca, M. Reinwald, W. Wegscheider, and C. Strunk, Phys. Rev. Lett. 92, 217001 (2004). 
8 Y. Blum, A. Tsukernik, M. Karpovski, and A. Palevski, Phys. Rev. Lett. 89, 187004 (2002).

9 C. Sürgers, T. Hoss, C. Schönenburger, and C. Strunk, J. Magn. Magn. Mater. 240, 598 (2002).

10 C. Bell, G. Burnell, C. W. Leung, E. J. Tarte, D.-J.Kang, and M. G. Blamire, Appl. Phys. Lett. 84, 1153 (2004).

11 A. A. Golubov, M. Y. Kupriyanov, and E. Il'ichev, Rev. Mod. Phys. 76, 411 (2004).

12 S. M. Frolov, D. J. Van Harlingen, V. A. Oboznov, V. V. Bolginov, and V. V. Ryazanov, Phys. Rev. B 70, 144505 (2004).

13 L. B. Ioffe, V. B. Geshkenbein, M. V. Feigel'man, A. L. Fauchère, and G. Blatter, Nature 398, 679 (1999).

14 J. E. Mooij, T. P. Orlando, L. Levitov, L. Tain, C. H. van der Wal, and S. Lloyd, Science 285, 1036 (1999).

15 Q. Yang, P. Holody, R. Loloee, L. L. Henry, W. P. Pratt Jr., P. A. Schroeder, and J. Bass, Phys. Rev. B 51, 3226 (1995).

16 W. Park, D. V. Baxter, S. Steenwyk, I. Moraru, W. P. Pratt Jr., and J. Bass, Phys. Rev. B 62, 1178 (2000).

17 P. Holody, W. C. Chiang, R. Loloee, J. Bass, W. P. Pratt Jr., and P. A. Schroeder, Phys. Rev. B 58, 12230 (1998).

18 R. Loloee, S. Urazhdin, W. P. Pratt Jr., H. Geng, and M. A. Crimp, Appl. Phys. Lett. 84, 2364 (2004).
19 T. Mühge, K. Theis-Bröhl, K. Westerholt, H. Zabel, N. N. Garif'yanov, Y. V. Goryunov, I. A. Garifullin, and G. G. Khaliullin, Phys. Rev. B 57, 5071 (1998).

20 T. Mühge, K. Westerholt, H. Zabel, N. N. Garif'yanov, Y. V. Goryunov, I. A. Garifullin, and G. G. Khaliullin, Phys. Rev. B 55, 8945 (1997).

21 C. Bell, E. J. Tarte, G. Burnell, C. W. Leung, D.-J.Kang, and M. G. Blamire, Phys. Rev. B 68, 144517 (2003).

22 C. Bell, R. H. Hadfield, G. Burnell, D.-J. Kang, M. J. Kappers, and M. G. Blamire, Nanotechnology 14, 630 (2003).

23 A. O. Adeyeye, J. A. C. Bland, C. Daboo, J. Lee, U. Ebels, and H. Ahmed, J. Appl. Phys. 79, 6120 (1996).

24 V. V. Ryazanov, Usp. Fiz. Nauk 169, 920 (1999), [Phys. Usp. 42825 (1999)].

25 D. Y. Petrovykh, K. N. Altmann, H. Höchst, M. Laubscher, S. Maat, G. J. Mankey, and F. J. Himpsel, Appl. Phys. Lett. 73, 3459 (1998).

26 Y. S. Barash and I. V. Bobkova, Phys. Rev. B 65, 144502 (2002).

27 J. Cayssol and G. Montambaux, Phys. Rev. B 71, 012507 (2005).

28 J. E. Dowman, M. L. A. MacVicar, and J. R. Waldram, Phys. Rev. 186, 452 (1969). 